\title{
Place navigation in the Morris water task: Effects of reduced platform interval lighting and pseudorandom platform positioning
}

\author{
BRYAN D. DEVAN, GRANT S. BLANK, and HERBERT L. PETRI \\ Towson State University, Towson, Maryland
}

\begin{abstract}
Two procedural components of place navigation training in the Morris (1981) water task were studied. Specifically, rats were required to escape onto a submerged (hidden) platform located in either a fixed or a pseudorandomly varied position throughout training. Following escape, the animals were either given unrestricted visual access to the extramaze environment for the duration of the platform interval $(45 \mathrm{sec})$ or denied this opportunity through reduced laboratory illumination. The results of the experiment indicate that (1) pseudorandom platform positioning primarily attenuated the rats' rate of acquisition rather than postasymptotic escape performance, and (2) visibility of distal cues from the goal location during training was not necessary for appropriate place navigation to occur. Further evidence obtained from a transfer test given on the final day of training suggests that rats trained with a pseudorandomly positioned platform may have acquired specific knowledge of multiple goal locations. These findings provide further support for the claim that rats acquire distal cue information primarily while en route to the platform.
\end{abstract}

The water-maze tasks developed by Morris (1981, 1984) have been used extensively to investigate the neurobiological bases of spatial learning in the rat (e.g., Hagen, Salamone, Simpson, Iverson, \& Morris, 1988; Kelsey \& Landry, 1988; Kolb, Sutherland, \& Whishaw, 1983; Lines \& Milner, 1985; Morris, 1989; Morris, Garrud, Rawlins, \& O'Keefe, 1982; Schenk \& Morris, 1985; Sutherland \& Rodriguez, 1988; Sutherland \& Rudy, 1988; Whishaw, 1985a; Whishaw \& Kolb, 1984; Whishaw, Mittleman, Bunch, \& Dunnett, 1987). It is easier to interpret the results of these and other studies involving some form of neural manipulation when the procedural and behavioral components of the tasks are well defined.

In the water-maze place task, rats are required to navigate to a hidden (i.e., slightly submerged) platform located in a large circular pool of clouded water. Although no local (intramaze) cues are present, rats are able to generate accurate trajectories to the submerged platform on the basis of its fixed spatial relation with distal (extramaze) room cues (Morris, 1981).

Following the rat's successful escape onto the submerged platform, the typical water-maze procedure requires that each rat remain there for a specific interval of time before the start of the next trial. However, the length of this interval (referred to here as the platform interval) has differed across experimental designs.

This research was supported by a Head Grant awarded to B. D. Devan. The authors thank Craig T. Johnson, Margaret L. Walker, Michael H. Figler, Clifton Santiago, and Robert Poh for their comments and assistance. Requests for reprints and/or further correspondence should be addressed to Herbert L. Petri, Department of Psychology, Towson State University, Towson, MD 21204.
Sutherland and Linggard's (1982) demonstration of latent spatial learning (see also Keith \& McVety, 1988; Whishaw, 1991) suggests that the platform interval may be a particularly important period during which rats acquire distal cue information. Furthermore, this claim is consistent with the pattern of rearing behavior observed during the platform interval. That is, rats tend to rear more frequently during the initial trials of place training (Sutherland, Whishaw, \& Kolb, 1983; Sutherland, Whishaw, \& Regehr, 1982; Whishaw \& Kolb, 1984), before performance (i.e., latency to escape) begins to asymptote. Sutherland and Dyck (1984) have suggested that this pattern of rearing behavior may represent a rat's active attempt to sample the extramaze configuration of distal room cues.

More recently, Sutherland, Chew, Baker, and Linggard (1987) obtained evidence that calls this interpretation into question. When Sutherland et al. reduced the level of laboratory illumination during select portions of a trial, they found that visual access to distal cues during the middle portion (i.e., while a rat was swimming to the platform) was necessary for the acquisition of place navigation. In contrast, restricted visibility of distal cues during the beginning of a trial or during the platform interval did not affect spatial performance.

Because former interpretations of platform learning have relied heavily on measures of rearing behavior, it may be of interest to know how this behavior relates to the more recent findings of Sutherland et al. (1987). If the time that a rat spends on the platform does not contribute significantly to place navigation, platform rearing should not show any consistent relationship to escape performance.

In addition, if, as Sutherland et al.'s (1987) findings suggest, rats obtain distal cue information while en route 
to the platform, performance may be facilitated in a condition that typically requires rats to swim more to locate the platform.

In the present experiment, rats were trained to navigate to a submerged platform located in either a fixed or a pseudorandomly varied position throughout training. Following escape, the laboratory lighting either was left on or was completely darkened for the duration of the platform interval. Measures of escape latency and rearing behavior were recorded during training, and a probe trial and a transfer test were included to assess the extent of acquired spatial knowledge.

\section{METHOD}

\section{Subjects}

Thirty-six experimentally naive male Long-Evans hooded rats, approximately 90 days of age (Charles River Laboratories), were used as subjects. The animals were housed in individual cages located in an experimental room that was maintained on a $12: 12$-h light:dark cycle (lights on $0700 \mathrm{~h}$ ) and kept at a temperature of $28^{\circ} \mathrm{C}$. All training and testing procedures described below were conducted during the light phase of the cycle. The animals were given ad-lib access to food and water.

\begin{abstract}
Apparatus
The water maze used in the experiment was a circular plywood tank $1.52 \mathrm{~m}$ in diameter and $0.45 \mathrm{~m}$ in height. The interior wall of the tank was lined with fiberglass and painted gray. Prior to training, the tank was filled to a depth of $26 \mathrm{~cm}$ with water. The water was maintained at a temperature of $27^{\circ} \mathrm{C} \pm 1^{\circ} \mathrm{C}$ and was rendered opaque by the addition of gray nontoxic water paint. A circular escape platform (12 cm in diameter and $24.5 \mathrm{~cm}$ in height) was constructed of plastic piping and was also painted gray.

A video camera was mounted on the ceiling directly above the water maze. This camera was connected to a video monitor and VHS recorder located in an adjacent room. An infrared scope was attached to a tripod and positioned at a fixed location within the laboratory. The infrared scope was used to observe instances of platform rearing during darkened laboratory conditions. The extramaze laboratory environment included several distal cues (e.g., a sink, bookshelves, posters, etc.), all of which were located at fixed positions.
\end{abstract}

\section{Procedure}

Each rat was handled (for 5 min on 3 consecutive days) and given two daily 80 -sec free-swim periods with the escape platform not present in the water maze. The animals were then randomly assigned to four independent treatment groups in a $2 \times 2$ factorial design, which combined two levels of platform interval lighting (lights on, or LOn, and lights out, or LOut) with two levels of platform positioning (fixed position, FP, and random position, RP). At the beginning of training, each of the four resulting groups contained 9 subjects. During training, however, 1 of the animals of group LOut/RP was excluded from the experiment (see below) and not replaced. (In condition LOn/FP, there were 9 subjects; in LOut/FP, 9; in LOn/RP, 9; and in LOut/RP, 8.)

Training. Place training was conducted over a 4-day period, during which time each rat received a total of 28 trials ( 8 trials on Days 1-3 and 4 trials on Day 4). A trial began with the rat's being placed in the water maze, facing the wall at one of four equidistant start points (arbitrarily designated NW, NE, SW, and SE) along the perimeter of the tank. Each start point was selected once within a block of four consecutive trials (i.e., block randomized).

After being released into the water, each rat was allowed to swim for a period of $80 \mathrm{sec}$ with the platform positioned in the center of one of four quadrants (N, S, E, and W). If a rat had not escaped onto the submerged platform within $80 \mathrm{sec}$, the experimenter gently guided it there by hand, and a latency score of $80 \mathrm{sec}$ was recorded. After escaping onto the platform, the animals were allowed to remain there for a 45 -sec platform interval. During this time, the appropriate lighting condition was employed, and the amount of time each rat spent in a rearing posture-operationally defined as occuring when the outstretched dorsal surface of the animal exceeded a $45^{\circ}$ angle from surface of the water-was recorded.

Following the completion of a trial, each rat was removed from the water tank and held for a brief period while the platform was pseudorandomly repositioned for the appropriate groups (i.e., LOn/RP and LOut/RP). For the groups trained with the platform located in a fixed position throughout training (LOn/FP and LOut/FP), the quadrant containing the platform was roughly counterbalanced (i.e., each of the four quadrants was chosen at least twice, with one quadrant being selected a third time within each FP group). During training, one animal in the LOut/RP group repeatedly left the platform during the 45 -sec interval following escape and was therefore excluded from the experiment. This animal was not replaced, and its data were not used in any analyses.

Testing. Immediately following Trial 28 of training, the platform was removed from the water tank, and each animal was given an $80-\mathrm{sec}$ probe test to assess the degree of spatial bias for the previous platform location(s). Two dependent measures of performance were recorded: (1) the amount of time spent in each of the four quadrants of the pool (quadrant time), and (2) the number of times an animal swam through an area that represented the circumference of the platform centered in each of the four quadrants (annulus crossings).

Following the completion of the probe test, the platform was repositioned in the water maze for four additional transfer test trials. For Groups LOn/FP and LOut/FP, the platform was located in the quadrant diagonally opposite to the training quadrant; for Groups LOn/RP and LOut/RP, the platform was located in a fixed position (pseudorandomly determined) for the first time. Both escape latency and rearing time were recorded during the transfer test.

\section{RESULTS}

The training data (both escape latency and rearing time) were analyzed with a $2 \times 2 \times 14$ lighting (LOn or LOut) $\times$ platform position (FP or RP) $\times$ blocks (of two trials) mixed factorial analysis of variance. A least squares procedure (Overall \& Spiegel, 1969) was used to adjust for unequal cell sizes.

The mean latencies to escape across blocks of two trials for each group are presented in Figure 1. Analysis of the latency data revealed a significant within-subject trial blocks effect $[F(13,403)=23.66, p<.001]$, indicating that all animals improved over the course of training. The between-subject lighting condition had no effect and did not interact with the other variables (all $F \mathrm{~s}<1.0$ ). A significant main effect of platform position $[F(1,31)=$ $31.42, p<.001]$ and a significant platform position $\times$ trial blocks interaction $[F(13,403)=2.02, p<.05]$ were obtained. However, a restricted analysis of the final day of training (Trial Blocks 13 and 14) failed to reveal any significant effects or interactions with the platform position variable (all $p s>.05$ ). This finding suggests that the primary difference between the FP and RP groups may be in their rates of acquisition rather than in their stabilized postasymptotic escape performance. In considering this result, one should note that the RP tasks involved es- 


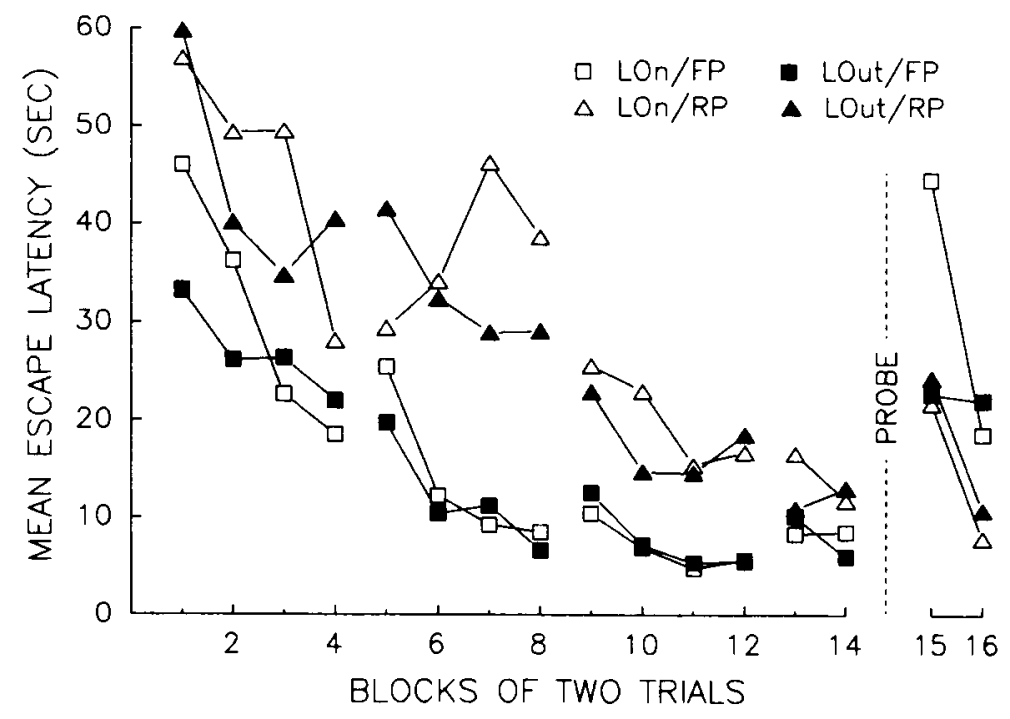

Figure 1. Mean latencies to escape onto the submerged platform for each group across blocks of two trials during training (Blocks 1-14) and the transfer test (Blocks 15 and 16).

caping onto the platform positioned at one of four locations; the lack of any significant difference between the RP and FP groups is quite impressive, given that no error measure (e.g., see Whishaw, 1985b) or correction term for equating task difficulty was used.

Figure 2 presents the mean rearing time for each group, as a function of trial blocks. Formal analysis of these data revealed a significant main effect of trial blocks $[F(13,403)$ $=21.75, p<.001]$ and platform position $[F(1,31)=$ $4.52, p<.05]$, and a lighting effect that approached significance $[F(1,31)=3.85, p=.06]$. There were no interactions among these variables $(p s>.10)$.
During the probe test (Trial 29), quadrant times and annulus crossings were measured to determine the degree of spatial bias for each quadrant. As shown in Figure 3, the groups trained with the platform located in a fixed position spent significantly more time in the training quadrant $[F(1,70)=342.88, p<.001]$ and made significantly more annulus crossings there $[F(1,70)=216.79, p<$ $.001]$ than in the three nontraining quadrants. In contrast, for the groups trained with the platform pseudorandomly varied from trial to trial, quadrant times and annulus crossings did not differ significantly as a function of quadrant or annulus position $(F s<1.0)$. Figure 4 (column B)

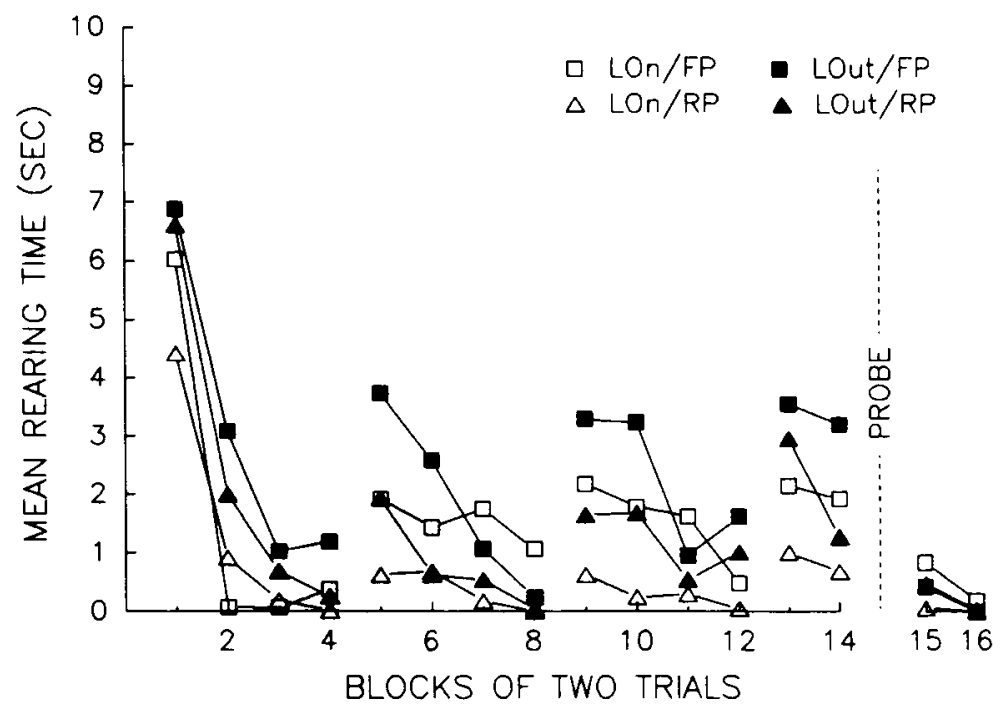

Figure 2. Mean time spent rearing on the platform for each group across blocks of two trials during training (Blocks 1-14) and the transfer test (Blocks 15 and 16). 

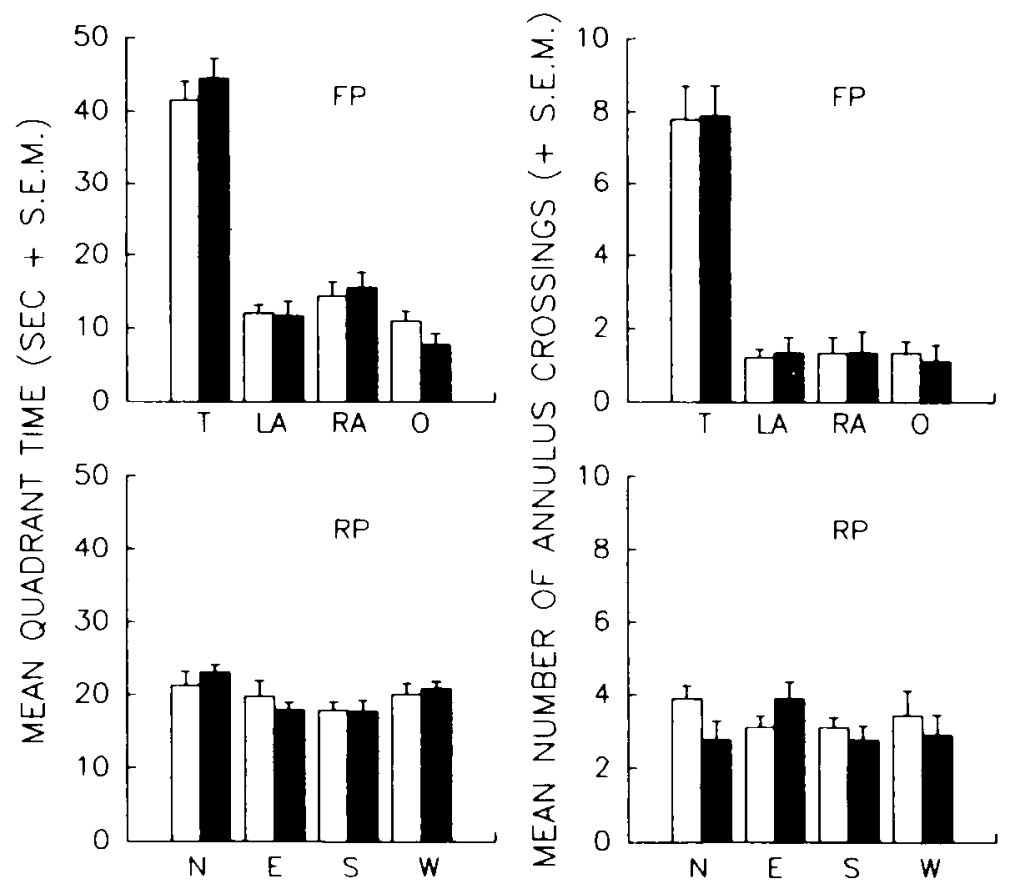

QUADRANT

Figure 3. Mean quadrant time (keft) and number of annulus crossings (right) as a function of quadrant position for groups trained with a fixed (top) or pseudorandomly positioned platform (bottom) during the probe test (Trial 29). FP, fixed position; RP, random position; $T$, training quadrant; $L A$, lef adjacent quadrant; $R A$, right adjecent quadrant; $O$, opposite quadrant; $N$, north quadrant; $E$, east quadrant; $S$, south quadrant; $W$, west quadrant. Open bars represent the means for the LOn groups, and solid bars represent the means for the LOut groups.

shows the swim paths taken by the median (or just below the median) rats in each group during the probe test. Note the restricted search patterns of Rat 23 (LOn/FP) andto a lesser extent-of Rat 28 (LOut/FP); this visual representation of spatial bias is not evident in the paths taken by Rats 31 (LOn/RP) and 36 (LOut/RP).

Analysis of variance of the escape latencies recorded during the transfer test (Trial Blocks 15 and 16; see Figure 1) revealed a significant main effect of trial blocks $[F(1,31)=22.67, p<.001]$ and platform position $[F(1,31)=5.15, p<.05]$. It is important to note that, in the latter effect, the RP groups escaped significantly faster than the FP groups, a trend opposite to that found during training. This result may, at least in part, be due to a persisting spatial bias for the former training quadrant in both FP groups (e.g., see Figure 4, column block C).

A significant trial block effect was found with the rearing measure taken during the transfer test $[F(1,31)=$ 9.72, $p<.001$; see Figure 2]; however, no other effects or interactions with this analysis of variance reached significance.

Former studies have shown that a dishabituation of rearing occurs when the platform is repositioned for transfer testing (Kolb et al., 1983; Sutherland et al., 1983; Suther- land et al., 1982; Whishaw, 1985a). In the present experiment, a comparison of the final trial block of training with the first transfer trial block (i.e., Block 14 with 15) revealed a significant decrease (i.e., habituation) in rearing time $[t(34)=2.80, p<.01$; see Figure 2]. However, the 80 -sec probe test conducted between training and transfer testing may have influenced the rearing shown on Trial Block 15.

By-trials Pearson product-moment correlation coefficients were calculated to assess the relationship between escape latency and rearing time. The results show nonsignificant correlations between these dependent measures during training $[r(978)=-.02, p>.40]$ and the transfer test $[r(138)=.11, p>.20]$ for all groups combined.

\section{DISCUSSION}

The results of the experiment indicate that rats are able to acquire spatial information when visual access to the extramaze environment (i.e., distal cues) is reduced during the platform interval. In addition, pseudorandom positioning of the platform during training primarily attenuated the rats' rate of acquisition rather than postasymptotic performance. 


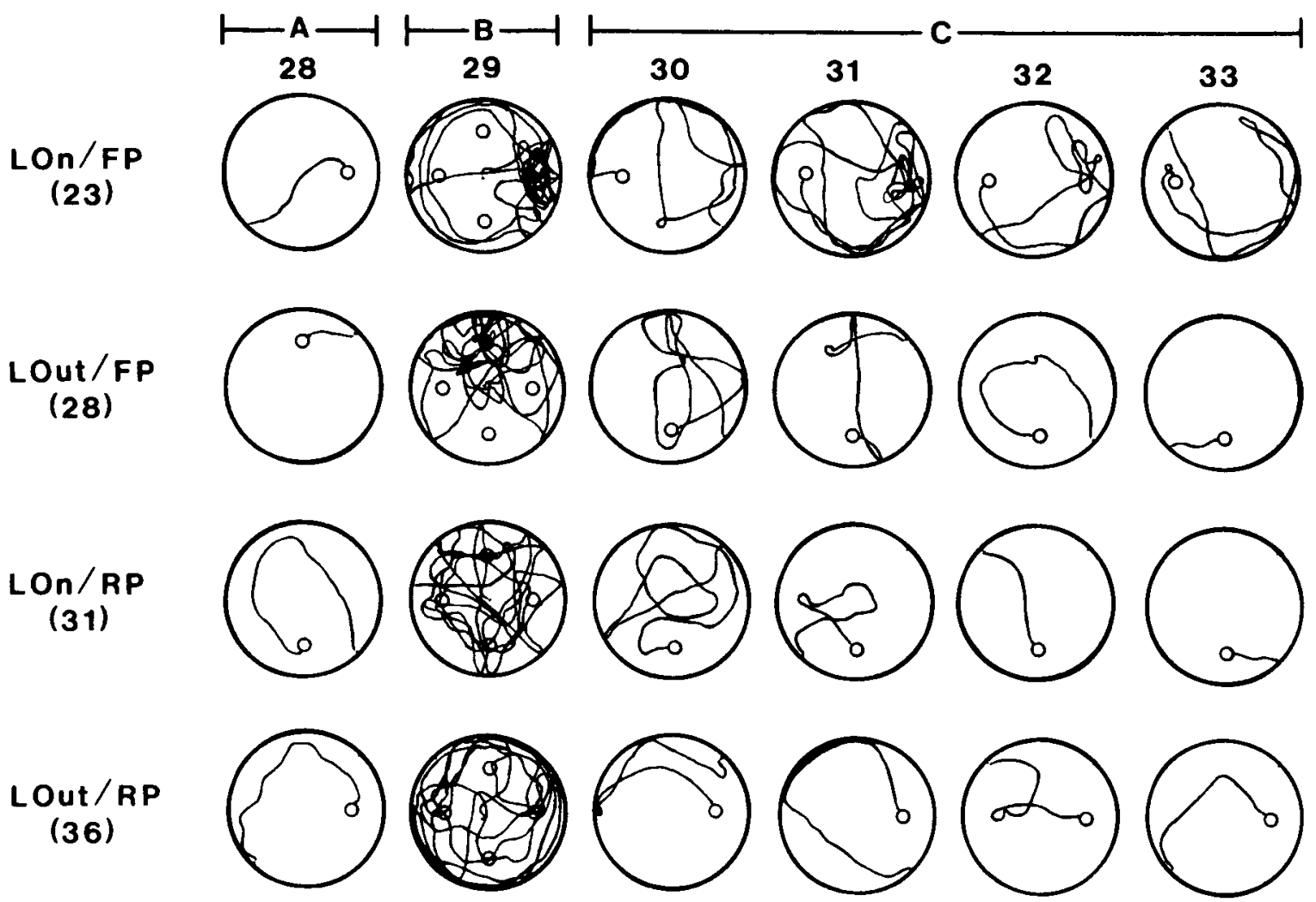

Figure 4. The swim paths taken by rats representing median or just below median performance (with respect to escape latency) in each group during (A) the final trial of training (i.e., Trial 28), (B) the 80-sec probe test (Trial 29), and (C) the transfer test (Trials 30-33).

The former finding provides a partial replication of Sutherland et al.'s (1987) second experiment, which involved a more detailed illumination design. The novel contribution made in the present study was the attempt to assess the role of platform rearing during normal and darkened laboratory conditions. Although slightly longer periods of rearing occurred during darkened conditions, this effect was not statistically significant. More importantly, our failure to confirm a dishabituation of rearing during the initial transfer trial block, particularly with the animals that were trained to locate a fixed platform, suggests that rearing behavior may not be related exclusively to distal cue sampling or to the integrity of place knowledge. Alternatively, platform rearing may be influenced by the proportion of time previously spent swimming (i.e., a fatigue factor), or the extent to which rearing is replaced by other relevant behaviors related to repeated exposure to the water (e.g., wet-dog shakes, grooming, and face washing). These explanations may account for the significant decrease in rearing time that occurred immediately following the 80-sec probe test (i.e., on the first two transfer trials). Furthermore, the low correlations obtained for the rearing and latency measures is consistent with this suggestion.

In support of the present findings, Lines and Milner (1985) have found that although rats with superior col- liculus lesions have reductions in orienting movements while on the platform, as well as severe spatial impairments (see also Milner \& Lines, 1983), the latter deficit is not the result of the former. Rather, the spatial impairments of the lesioned animals were attributable to a disturbance of ambient vision that occurred during swimming.

In his initial investigation, Morris (1981) found that the swim paths of Group "Place-Random" (corresponding here to Group LOn/RP) lacked any directionality, and suggested that this group should therefore serve as a control for assessing the more effective escape performance of Group "Place." Although the directional behavior of the random group is, by definition, at chance levels, their knowledge of multiple platform locations may have been underestimated previously.

In the present design, RP place training was conducted over 28 trials, during which time an 80-sec trial termination interval was employed. In Morris's (1981) investigation, only 20 trials of training were given, and the trial termination interval was set at $60 \mathrm{sec}$. These procedural variations may account for the improved performance of the RP groups during the latter trials of training in the present study.

Sutherland et al. (1987) have shown that rats that are afforded unrestricted swimming and viewing access to the extramaze environment while en route to the platform are 
able to generate accurate trajectories to the platform when started from novel locations. In addition, Sutherland et al. identified the middle segment of a trial (i.e., when a rat is swimming to the platform) as the period during which rats acquire distal cue information. Because both RP groups included in the present investigation were allowed unrestricted swimming and viewing access for an extended period $(80 \mathrm{sec})$ during the middle portion of a trial, it seems plausible that they may have acquired some distal cue information over the course of training.

This suggestion is consistent with the results of the transfer test. That is, when both RP groups were given the opportunity to escape onto the submerged platform newly located in a fixed position for four consecutive trials, their escape performance surpassed that of the FP groups (see Figure 1), which were at this time required to locate the platform repositioned in the quadrant diagonally opposite to the training quadrant. Essentially, Morris obtained similar results with his transfer test (Morris, 1981, Figure 8, p. 251), and he acknowledged that Group "Place-Random" showed "no obvious sign that it had learned to ignore place cues"' (p. 250). The remaining question, then, is whether the RP groups were able to use their knowledge of distal cues to navigate during training.

Inspection of the swim paths taken by representative animals from each group on the final trial of training (Figure 4, column A) suggests that the RP rats were employing a taxon strategy (O'Keefe \& Nadel, 1978), which was exemplified by a looped pattern that passed through the center of multiple quadrants in the present experiment. Given the random nature of the task, this strategy would seem, on the average, to provide the RP animals with a more efficient means of escape than would swimming directly to individual annuli located in opposing quadrants.

The demonstration of apparent taxon strategy usage, however, does not indicate the specific knowledge an animal has acquired, nor does it rule out the potential for making other types of responses. Indeed, several studies have shown that rats may make simultaneous use of several search strategies while navigating in the water maze (Schenk \& Morris, 1985; Sutherland \& Dyck, 1984; Whishaw, 1985a; Whishaw \& Mittleman, 1986). Thus, the overt expression of a particular strategy may be more a function of the experimental parameters employed within a study than a representation of the quality and extent of acquired knowledge.

Olton (1979) has described the difficulty in determining the degree to which a taxon or spatial mapping system has been involved in the generation of a direction strategy. In addition, as Olton points out, most maze tasks that are thought to require a location strategy are also solvable by a direction strategy. If the results obtained here (particularly during the transfer test) provide any indication that Group LOn/RP acquired specific location information during training, as we suggest, then the water- maze "Place-Random" task may be considered a potential "location without direction" problem. Such a task may prove useful for testing the predictions of spatial/ mnemonic theories of hippocampal function (e.g., O'Keefe \& Nadel, 1978; Olton, Becker, \& Handelmann, 1979; Sutherland \& Rudy, 1989), because the ambiguity associated with directionality is eliminated by pseudorandomly varying the position of the hidden platform across training trials.

In addition to the potential usefulness of the "PlaceRandom" task, the present results provide one caveat for future water-maze investigations in which the use of lesion techniques or pharmacological agents would necessitate the inclusion of control procedures. That is, because our findings indicate that rats may indeed acquire spatial information when the goal platform is pseudorandomly varied from trial to trial (see also Whishaw \& Mittleman, 1986), the "Place-Random' task should not be considered an appropriate control condition for distinguishing between pure spatial deficits and those attributable to sensory, attentional, motor, or motivational impairments. What may prove to be of more value in identifying spatial deficits is the use of probe trials and/or transfer tests to further identify and characterize all relevant strategies acquired through training. We believe that an emphasis on the latter procedures may offer greater insight into the complex spatial navigation behavior of rats.

\section{REFERENCES}

Hagen, J. J., SAlamone, J. D., Simpson, J., Iverson, S. D., \& Morris, R. G. M. (1988). Place-navigation in rats is impaired by lesions of medial septum and diagonal band but not nucleus basalis magnocellularis. Behavioural Brain Research, 27, 9-20.

KeITH, J. R., * MCVETY, K. M. (1988). Latent place learning in a novel environment and the influences of prior training in rats. Psychobiology, 16, 146-151.

KelSEY, J. E., \& LANDRY, B. A. (1988). Medial septal lesions disnupt spatial mapping ability in rats. Behavional Neunoscience, 102, $289-293$.

Kolb, B., Sutherland, R. J., \& Whishaw, I. Q. (1983). A comparison of the contributions of the frontal and parietal association cortex to spatial localization in rats. Behavioral Neuroscience, 97, 13-27.

Lines, C. R., Milner, A. D. (1985). A deficit in ambient visual guidance following superior colliculus lesions in rats. Behavioral Neuroscience, 99, 707-716.

Milner, A. D., Lines, C. R. (1983). Stimulus sampling and the use of distal visual cues in rats with lesions of the superior colliculus. Behavioural Brain Research, 8, 387-401.

Morris, R. G. M. (1981). Spatial localization does not require the presence of local cues. Learning \& Motivation, 12, 239-260.

MorRis, R. G. M. (1984). Developments of a water-maze procedure for studying spatial learning in the rat. Journal of Neuroscience Methods, 11, 47-60.

MoRRIS, R. G. M. (1989). Synaptic plasticity and learning: Selective impairment of learning in rats and blockade of long-term potentiation in vivo by the N-methyl-D-aspartate receptor Antagonist APS. Journal of Neuroscience, 9, 3040-3057.

Morris, R. G. M., Garrud, P., Rawlins, J. N. P., O'Keefe, J. O. (1982). Place navigation impaired in rats with hippocampal lesions. Nature, 297, 681-683.

O'KeEFE, J. O., NADEL, L. (1978). The hippocampus as a cognitive map. Oxford: Oxford University Press, Clarendon Press. 
OLton, D. S. (1979). Inner and outer space: The neuroanatomical bases of spatially organized behaviors. Behavioral \& Brain Sciences, 2 , 511-512.

Olton, D. S., Becker, J. T., \& Handelmann, G. E. (1979). Hippocampus, space, and memory. Behavioral \& Brain Sciences, 2, 313-365.

Overall, J. E., SPiegel, D. K. (1969). Concerning least squares analysis of experimental data. Psychological Bulletin, 84, 311-322.

Schenk, F., \& MoRrIs, R. G. M. (1985). Dissociation between components of spatial memory in rats after recovery from the effects of retrohippocampal lesions. Experimental Brain Research, 58, 11-28.

Sutherland, R. J., Chew, G. L., Baker, J. C., LingGard, R. C. (1987). Some limitations on the use of distal cues in place navigation by rats. Psychobiology, 15, 48-57.

Sutherland, R. J., Dyck, R. H. (1984). Place navigation by rats in a swimming pool. Canadian Journal of Psychology, 38, 322-347.

Sutherland, R. J., LingGard, R. (1982). Being there: A novel demonstration of latent spatial learning in the rat. Behavioral \& Neural Biology, 36, 103-107.

Sutherland, R. J., Rodriguez, A. J. (1988). The role of the fornix/fimbria and some related subcortical structures in place learning and memory. Behavioural Brain Research, 32, 265-277.

Sutherland, R. J., \& Rudy, J. W. (1988). Place learning in the Morris place navigation task is impaired by damage to the hippocampal formation even if the temporal demands are reduced. Psychobiology, 16, 157-163.

Sutherland, R. J., \& Rudy, J. W. (1989). Configural association theory: The role of the hippocampal formation in learning, memory, and amnesia. Psychobiology, 17, 129-144.

Sutherland, R. J., Whishaw, I. Q., KolB, B. (1983). A behavioural analysis of spatial localization following electrolytic, kainate- or colchicine-induced damage to the hippocampal formation in the rat. Behavioural Brain Research, 7, 133-153.

Sutherland, R. J., Whishaw, I. Q., \& Regehr, J. C. (1982). Cholinergic receptor blockade impairs spatial localization by use of distal cues in the rat. Journal of Comparative \& Physiological Psychology, 96, 563-573.

WHISHAW, I. Q. (1985a). Cholinergic receptor blockade in the rat impairs locale but not taxon strategies for place navigation in a swimming pool. Behavioral Neuroscience, 99, 979-1005.

WHISHAW, I. Q. (1985b). Formation of a place learning-set by the rat: A new paradigm for neurobehavioral studies. Physiology \& Behavior, 35, 139-143.

Whishaw, I. Q. (1991). Latent learning in a swimming pool place task by rats: Evidence for the use of associative and not cognitive mapping processes. Quarterly Joumal of Experimental Psychology, 43B, 83-103.

Whishaw, I. Q., Kolв, B. (1984). Decortication abolishes place but not cue learning in rats. Behavioural Brain Research, 11, 123-134.

Whishaw, I. Q., Mittleman, G. (1986). Visits to starts, routes, and places by rats (Rattus norvegicus) in swimming pool navigation tasks. Journal of Comparative Psychology, 100, 422-431.

Whishaw, I. Q., Mittleman, G., Bunch, S. T., Dunnett, S. B. (1987). Impairments in the acquisition, retention and selection of spatial navigation strategies after medial caudate-putamen lesions in rats. Behavioural Brain Research, 24, 125-138.

(Manuscript received October 23, 1991; revision accepted for publication February 5, 1992.) 